\title{
Quebec doctors aim to fill marijuana knowledge gaps
}

$\mathrm{D}$ octors in Quebec who prescribe medical marijuana will automatically take part in a province-wide research project to assess the risks and benefits of the drug.

Health Canada recently shifted responsibility for deciding who should have access to the drug onto the shoulders of individual doctors, raising concerns among physician groups, including the Canadian Medical Association, about the lack of robust data on the safety and effectiveness of marijuana used for medical purposes.

"The new regulations do not address the key concern for our physicians and surgeons: the shortage of evidencebased research that conclusively supports the safety, risks and benefits of medical marijuana," said Tom McMillan, a spokesman for the Royal College of Physicians and Surgeons of Canada.

The research project, run by the Collège des médecins du Québec and the Canadian Consortium for the Investigation of Cannabinoids (CCIC), is mandatory for all Quebec doctors who prescribe marijuana. The college's rules state that any unrecognized treatment can only be used within a research framework.

"There is clearly a lack of scientific knowledge about the use of cannabis," said Dr. Yves Robert, secretary of the

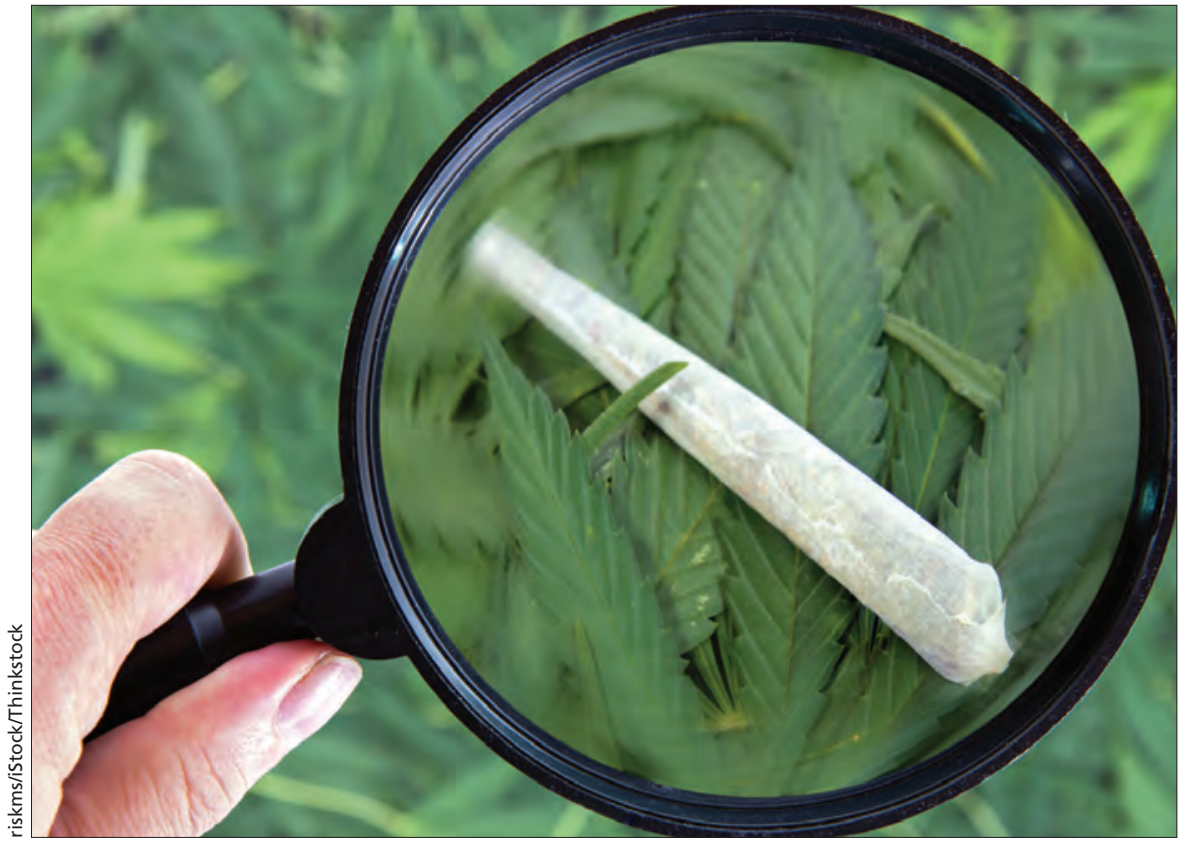

As part of a pharmacovigilance study, physicians will provide data on patients and their diagnoses, how the prescribed marijuana is used and at what dosage, as well as the effects of the drug - both good and bad.

to test a hypothesis," says Robert. This is the type of monitoring that would normally be done by Health Canada for an officially licensed drug.

Dr. Mark Ware, executive director of the CCIC, said that although the college's project can't measure the efficacy of cannabis in a clinical sense, the systematic data on patients and out-

\section{Quebec doctors who prescribe marijuana must take part in the research.}

college, "so we thought this was the best path to take in order to provide more expertise and scientific grounds for physicians and more safety for patients."

The research project is a pharmacovigilance study in which physicians will provide data on patients and their diagnoses, how the prescribed marijuana is used and at what dosage, as well as the effects of the drug - both good and bad. "It's really a surveillance program rather than research that tries comes will help to develop guidance for clinicians about the kinds of things to look for at screening and what safety issues may be pertinent. "So we can actually inform the process, rather than having patients using cannabis under the legal framework and we learn nothing."

Existing studies of medical marijuana tend to be lab-based, using a standardized product on carefully selected subjects, said Robert. And a study in the Netherlands, where the drug is dis- pensed through pharmacies, looked at dosages prescribed, but did not include any data on patient outcomes, Ware added. A major benefit of the Quebec study is that it is population-based and will collect data on the myriad strains and products on offer from licensed suppliers.

With an estimated 3000 patients using medical marijuana in Quebec, Ware expects that within two to three years the volume of data collected will be sufficient to draw some conclusions about safety and effectiveness, and potentially scale back the research project.

Robert says that while there are no plans at present to expand the research program to physicians in other provinces, he would like the information gathered in Quebec to be shared more widely. Ware said that with Quebec acting as a pilot, he sees no reason why the CCIC could not open it up to the rest of Canada. - Brian Owens, St. Stephen, NB

CMAJ 2014. DOI:10.1503/cmaj.109-4791 